\title{
Reseña del libpo The Moral Psychology of Hope de Claudia Blöser y Titus Stahl (2019)*
}

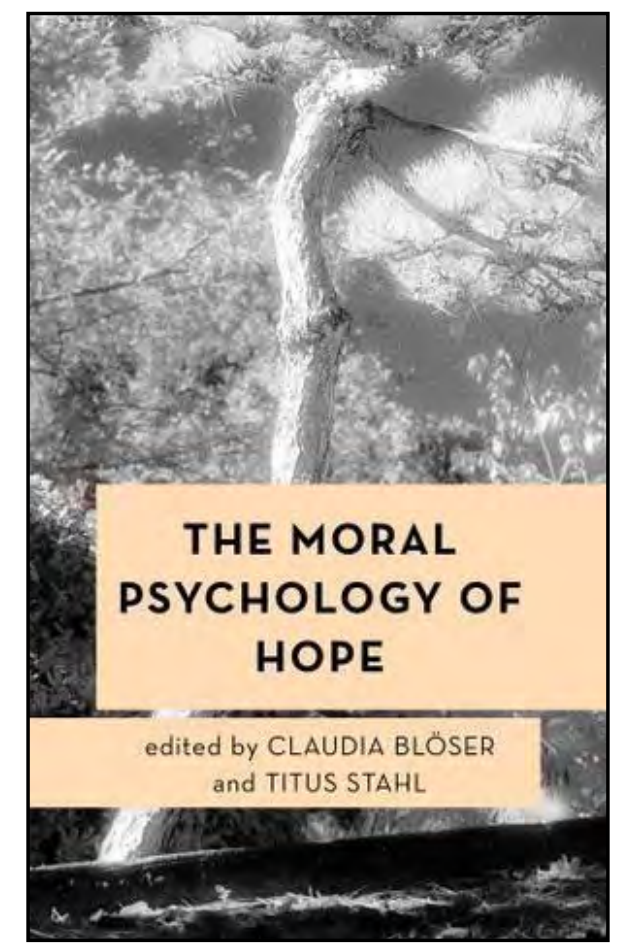

Portada del libro The moral psychology of hope.

Fuente: Blöser y Sthal (2019).

\author{
Roberto Carlos Dávila Morán \\ Universidad Privada del Norte (UPN), Lima, Perú \\ rdavila430@gmail.com \\ https://orcid.org/0000-0003-3181-8801
}

Cómo citar: Dávila, R. (2021). Reseña del libro The Moral Psychology of Hope de Claudia Blöser y Titus Stahl. Ciencias Sociales y Educación, 10(20), 317-326. https://doi.org/10.22395/csye.v10n20a15 Recibido: 8 de mayo de 2021

Aprobado: 8 de junio de 2021 
Claudia Blöser y Titus Stahl han reunido un volumen amplio sobre la naturaleza, la historia y el significado social de la esperanza. El volumen no escatima en historia; seis de sus quince capítulos se centran en la historia filosófica de la esperanza, y otros incluyen una discusión extensa de figuras históricas y tradiciones. El volumen también hace contribuciones a nuestra comprensión de la naturaleza de la esperanza, considerada tanto como una actitud como un rasgo (quizás una virtud) del carácter. El volumen se cierra con una sección sobre los contextos sociales de la esperanza, donde se presta atención a temas como el papel de la esperanza en la justificación política y la racionalidad de esperar una prosperidad humana generalizada ante un posible colapso ambiental. Es un volumen rico. Tendré ocasión a continuación para tocar cada uno de sus capítulos.

Las últimas dos décadas aproximadamente de discusión filosófica sobre la actitud de esperanza pueden caracterizarse por un cambio desde la "visión estándar", según la cual la esperanza se compone de i) la creencia de que algún resultado o estado de cosas $\mathrm{X}$ es posible pero no garantizado, y ii) el deseo de que $\mathrm{X}$ sea el caso. La visión estándar, se sostiene generalmente, no sólo falla en proporcionar condiciones suficientes para la esperanza; parece, de manera más condenatoria, contar los casos de desesperación, lo opuesto de la esperanza, como ejemplos de esperanza. Porque la desesperación también implica intuitivamente creencias y deseos del tipo anterior (de hecho, como varios han señalado, parece que dos agentes pueden asignar la misma probabilidad a algún resultado y desear ese resultado en el mismo grado, pero uno se llena de esperanza y el otro de desesperación). Actualmente se sostiene ampliamente que se debe agregar algún componente adicional, además de la fe y el deseo, para procurar las condiciones suficientes para la esperanza. Una forma útil de caracterizar lo que estas propuestas recientes tienen en común se encuentra en el capítulo de Martin (2019), donde escribe:

Recientemente ha surgido un consenso aproximado de que la esperanza tiene tres componentes principales: primero, la creencia de que el resultado esperado es posible pero no garantizado; segundo, un deseo o preferencia por ese resultado; y una tercera cosa que equivale a una actitud positiva de "qué pasaría si" hacia un futuro que contenga ese resultado. (Blöser y Titus, 2019, p. 231-232)

En contraste con el agente desesperado, que puede desear $\mathrm{X}$ y creer que $\mathrm{X}$ es posible pero no garantizado, el agente que espera tiene una actitud positiva de "qué pasaría si" con respecto a la obtención de X. La visión en marcha, en otras palabras, refleja que, en contraste con la desesperación, la esperanza implica una orientación positiva hacia la posibilidad de lo que se desea.

Stockdale (2019) desafía esta idea. En particular, el capítulo de Stockdale desafía la idea de que, en la medida en que la esperanza implica emoción, implica una emoción positiva. Stockdale (2019) sostiene que la atención a las condiciones 
sociales no ideales revela que hay una especie de esperanza pasada por alto y con valencia negativa. Este tipo de esperanza, "esperanza temerosa" (p. 117), como la etiqueta Stockdale (2019), no sólo se asocia con, sino que se constituye por miedo. Su tono afectivo es totalmente negativo. Stockdale (2019) proporciona, en apoyo de su propuesta, un ejemplo en el que una mujer afroamericana llamada Kayla es detenida sin razón aparente y experimenta una actitud de valencia negativa cuando el oficial se acerca a su automóvil. Stockdale (2019) caracteriza la actitud de Kayla como una "terrible esperanza" de que "el oficial de policía con el que estoy a punto de encontrarme no utilice la violencia hacia mí" (p. 125). Sin embargo, dado que Kayla representa al oficial como "amenazante", no está claro por qué no caracterizaríamos la respuesta de Kayla, más simplemente, como una de miedo. Después de todo, el miedo por algún posible estado de cosas implica ver ese estado de cosas como amenazante o peligroso. Stockdale describe a Kayla actuar violentamente hacia ella. Pero, ¿no podemos conformarnos con atribuirle a Kayla la respuesta más familiar (es decir, una dirigida no hacia la omisión sino hacia la acción) de miedo a que el oficial se comporte violentamente con ella? La palabra esperanza se puede emplear típicamente para describir a la persona que experimenta miedo: incluso la persona que huye con miedo del perro amenazante, por ejemplo, puede ser descrito como "esperando escapar del perro" (p. 128). Sin embargo, no está claro si este hecho lingüístico autoriza una visión de la economía mental de estos agentes temerosos en el sentido de que incluye una actitud de esperanza temerosa además de la de miedo, especialmente porque la naturaleza del miedo orientada hacia el futuro está preparada para dar cuenta de estos factores. pensamientos de los agentes sobre la seguridad. Estas preocupaciones sobre la adecuación descriptiva y la recompensa psicológica moral pueden ser especialmente importantes dado que la visión de Stockdale (2019) tiene el costo de rechazar una explicación unificada de la esperanza como en parte constituida por una perspectiva positiva.

El capítulo de Benton (2019) presenta a los lectores la relación entre conocimiento y esperanza. Benton proporciona apoyo lingüístico a la tesis de que espera que $\mathrm{p}$ sea incompatible tanto con el conocimiento que p como con el conocimiento que no p; cuando se espera que p, no se sabe si p. Benton (2019) pasa en la última parte del capítulo en cuestiones relativas a la racionalidad de continuar con la esperanza frente a la creciente evidencia en contra de la obtención del resultado esperado. Como señala Benton (2019), a veces se alaba al persistente que espera por su "firmeza", mientras que en otras ocasiones se burlan de él por "no ser realista». Benton propone que la racionalidad de perseverar en la esperanza, a pesar de la creciente evidencia contraria al resultado esperado, dependerá de la capacidad de recuperación que uno pueda 
anticipar frente a una mayor decepción. Como tal, la racionalidad de perseverar en la esperanza depende tanto de juicios teóricos como de juicios prácticos.

¿Is Hope a Moral Virtue? de Snow (2019) responde afirmativamente a la pregunta del título, agregando a sus puntos de vista previamente publicados que la esperanza puede ser tanto una virtud intelectual como una virtud cívica democrática. Snow desarrolla una descripción ampliamente aristotélica de la esperanza como virtud moral, prestando especial atención al desarrollo de la esperanza como virtud natural a través de la esperanza como virtud moral, donde una marca de esta última es que está guiada por la sabiduría práctica. Para Snow, la esperanza tiene una conexión no accidental con una orientación agencial positiva, ya que "tener esperanza es parte de una perspectiva positiva y progresista que está abierta a posibilidades futuras y posiciona a la persona esperanzada para involucrar sus habilidades cognitivas, afectivas, imaginativas y capacidades agenciales en los esfuerzos por obtener bienes futuros" (p. 180). Al desarrollar una visión de la esperanza como virtud moral.

Pessimism and the Possibility of Hope de Vice (2019) defiende la compatibilidad de una disposición esperanzadora con el pesimismo, donde el pesimismo se entiende como "la perspectiva o actitud sobre el mundo humano que es escéptica de la posibilidad de un progreso moral significativo, y que considera el daño que los seres humanos causan es moralmente más destacado y de mayor peso que el bien que provocan" (p. 160). Partiendo de Gabriel Marcel, Vice se une a quienes entienden la esperanza (o, mejor dicho, la ilusión) como una orientación agencial positiva, aquella desde la que "el futuro se considera abierto y receptivo a nuestros esfuerzos" (p. 169). Así interpretada, la esperanza encuentra su opuesto en la desesperación más que en el pesimismo. Se presta especial atención a las diferencias en la fenomenología temporal entre la esperanza y la desesperación. A la desesperación, según Vice, capitular, mientras que esperar es negarse a capitular. La esperanza se caracteriza de esta manera como una forma de paciencia (una idea coherente con las recomendaciones en el sentido de que ino nos rendimos y nos aferramos a la esperanza). Curiosamente, Vice construye la esperanza en términos estéticos positivos, escribiendo que hay una "flexibilidad y gracia en la negativa a capitular".

Hope in Contemporary Psychology, de Gallagher, D'Souza, y Richardson (2019), se centra en el influyente modelo de esperanza desarrollado por Snyder, según el cual la esperanza elige un rasgo que implica verse a uno mismo como agente eficaz. Es decir, en el modelo dominante:

La esperanza es un rasgo cognitivo doble que representa la capacidad de identificar estrategias para lograr los objetivos propios (es decir, el pensamiento de caminos) y tener la motivación y la determinación para implementar con éxito esos 
caminos (es decir, el pensamiento de agencia) para lograr los objetivos propios.

(Snyder, 2002 ; Snyder et al., 1991, p. 192)

Aquellos "altos contenidos de esperanza" — según varias escalas de medida (que se señala en el capítulo) desarrollado por Snyder y otros - se distinguen de los de alto contenido optimismo en que los altos hopers creen no sólo que sus objetivos serán satisfechos, pero que estarán satisfechos a través de su propia agencia. El capítulo también analiza la evidencia de varias intervenciones terapéuticas basadas en la esperanza, incluidas aquellas que parecen tener cierta efectividad para reducir los síntomas de depresión y ansiedad.

Interpersonal Hope, Martin (2019) presenta una visión del tipo de esperanza que las personas invierten en los demás -esperanza interpersonal-proporcionando a lo largo del camino una taxonomía general de la esperanza. En la concepción de esperanza de "agencia socialmente extendida" (p. 233) desarrollada por Martin, «invertir esperanza en una persona es esperar extender la agencia de uno a través de la suya" (p. 237). El ejemplo paradigmático de Martin es aquel en el que un padre invierte en su hija la esperanza de una vida mejor. La esperanza interpersonal del padre consiste en la esperanza de extender su agencia a través de su hija, lo que se hace proporcionando a su hija, la participada, "recursos de agencia" (p. 240). Estos incluyen no solo los recursos materiales, sino el trabajo involucrado en la crianza y educación de un niño, junto con los recursos emocionales y motivacionales. El relato que se ofrece es rico y el capítulo puede ser de especial importancia para aquellos interesados en la psicología moral de las actitudes de los participantes más allá de la tan discutida tríada de resentimiento, indignación y culpa. Martin desarrolla su punto de vista con la mirada puesta en la gama de respuestas interpersonales que tenemos cuando las esperanzas que invertimos en los demás se frustran o se realizan.

Si bien creo que Martin (2019) discute un fenómeno normativo importante y pasado por alto, me pregunto si la centralidad otorgada a la contribución agencial del aspirante corre el riesgo de dejar de lado ejemplos de esperanza interpersonal. Parecemos capaces de poner esperanza en agentes con los que no estamos personalmente comprometidos y a quienes no podemos extender nuestra agencia de la manera demostrada por el padre. Supongamos, por ejemplo, que Belle es una ermitaña que vive en las montañas, está equipada con televisión por satélite y le apasiona el patinaje artístico. En los últimos años, Belle ha desarrollado un interés en Alicia, una patinadora artística en ciernes. Belle se deja llevar por la gracia de Alicia sobre el hielo y ve su considerable potencial. Quizás Belle y Alicia también comparten un origen nacional, donde la nación no está representada en patinaje artístico al nivel de Alicia. Supongamos que Alicia está a punto de actuar en sus primeros Juegos Olímpicos de invierno; 
Belle observa con emoción y nerviosismo. Aunque Belle no lo hace contribuir con recursos de agencia del tipo que podrían proporcionar sus amigos, familiares, entrenadores e incluso otros fanáticos (a través de animar en las gradas, comprar mercadería), sin embargo, parece plausible que Belle pueda invertir la esperanza en Alicia (por ejemplo, para triunfar como patinador, para seguir desarrollándose). Martin puede decir que Belle está involucrada emocionalmente en Alicia, y esto seguramente es correcto, pero entender esto como un caso de agencia socialmente extendida parece, en el mejor de los casos, tenso. Al discutir casos similares, Martin afirma que puede ser razonable "que los fanáticos de las celebridades atléticas o políticas se sientan decepcionados por las malas actuaciones o las fallas en la defensa de los valores los fans sentían que tenían en común" (p. 245). Esto suena muy bien, pero parece apuntar en la dirección de una visión de esperanza interpersonal centrada en algo como compartir el valor, lo que podría hacerse desde lejos. - en lugar de la extensión de la agencia.

Paso ahora a los capítulos históricamente centrados en el volumen. En Hope in Archaic and Classical Greek, Cairns (2019) defiende la tesis mesurada de que, aunque la palabra griega elpis a veces significa más o menos lo que queremos decir con "esperanza", el rango semántico de elpis es demasiado amplio para mapear claramente nuestro concepto de esperanza. Después de argumentar a favor de esta tesis a través de Aristóteles y Platón, Cairns recurre a la poesía griega clásica, donde elpis se interpreta como una deidad capaz de proporcionar sustento espiritual pero que ocasionalmente prepara agentes para las frustraciones que acompañan a luchar por lo inalcanzable. Elpis de esta manera opera dentro de una cosmovisión en la que las aspiraciones humanas deben mantenerse bajo control mediante una apreciación de hasta qué punto nuestros éxitos y alegrías dependen de una miríada de factores que escapan a nuestro control.

Hope in Christianity, Jeffrey (2019) sostiene que se pueden distinguir dos tipos de esperanza en un relato cristiano: i) esperanza dirigida hacia eventos posibles, y ii) esperanza en Dios. Este último se caracteriza por incluir el deseo de unión con Dios, donde no debe entenderse como una especie de esperanza para la obtención de algún evento. Es decir, la esperanza dirigida por Dios es irreductible a la esperanza dirigida por eventos, en parte, sostiene Jeffrey, porque la esperanza dirigida por Dios no necesita tener un objeto determinado. Jeffrey ofrece como ejemplo la esperanza que Abraham deposita en Dios cuando deja su hogar por mandato de Dios. En ese punto, no sabe qué esperar de Dios (p. 45). Sin embargo, no está claro por qué no deberíamos caracterizar a Abraham en lugar de confiar en Dios, porque la confianza parece tomar más cómodamente a un agente como su objeto sin tomar también algún evento / acción adicional como su objetivo. De interés potencial para quienes consideran los aspectos epistemológicos de la esperanza es que a veces se entiende que la esperanza 
va acompañada de una especie de certeza no probatoria, alcanzada por la fe, donde "la fe consiste en la adhesión segura a una proposición con certeza, pero sin una comprensión completa" (p. 50).

Hope in Kant, Blöser (2019) desarrolla una descripción unificada de la esperanza como se analiza en una variedad de obras de Kant (la primera y la segunda crítica, la religión y los escritos políticos de Kant). Una suposición clave de la visión presentada es que la esperanza presupone que se considera que algún fin está más allá del poder de uno para realizarlo. Pero mientras esta presuposición distingue la esperanza de la elección, para distinguir la esperanza del desear, es necesaria una segunda suposición clave, a saber, la presuposición de que el fin tiene fundamentos, es decir, que puede ser provocado, a través de algún poder. Como sostiene además Blöser, mientras Kant sostiene que carecemos de conocimiento de los fundamentos de la esperanza, la esperanza puede ser racional en la medida en que sea racional tener fe o "creencia moral" en los fundamentos de la propia esperanza. Una de esas creencias morales que podemos sostener racionalmente, según Kant, es que en la existencia de Dios y una vida después de la muerte, la ley moral ordena que actuemos de tal manera que seamos merecedores de la felicidad, pero el mundo presente no es uno en el que la felicidad está garantizada por una acción moralmente digna. Según la lectura de Blöser, Kant presenta un argumento estructuralmente paralelo a favor de la racionalidad de la esperanza de una paz perpetua. Tenemos el "deber de promover y, por lo tanto, aproximarnos a la paz perpetua" (p. 63), por lo que podemos esperar la paz perpetua, pero como no podemos asumir que podemos lograr este fin por nuestra cuenta, esta esperanza presupone la fe en su fundamento, es decir, en el orden providencial. Además de identificar posibles debilidades en los diversos argumentos de Kant, Blöser atiende la cuestión de si la esperanza ocupa un papel motivacional distinto en la psicología moral de Kant.

El capítulo de Fremstedal (2019), Kierkegaard on hope as essential to the selfhood (p. 83), avanza en la afirmación de que Kierkegaard sostiene que una identidad práctica coherente requiere no solo, como otros han argumentado, un compromiso incondicional con un ideal moral sino también la "esperanza de que nuestros ideales sean realizables" (p. 90). Una característica extraña de la opinión es que parece ser una cuestión de estipulación que la desesperación implica una doble mentalidad y, por lo tanto, excluye una identidad práctica coherente. Puede ser que este lector simplemente requiera una mayor familiaridad con Kierkegaard sobre la desesperación, ya que, como lo discutió Fremstedal, es un compromiso metodológico de Kierkegaard que su explicación de la esperanza se desarrolle vía negativa, trabajando hacia arriba, por así decirlo, desde la desesperación. 
Antes de apartarme de la filosofía europea del siglo XIX, observo que, en varios lugares del volumen, incluida la introducción de los editores y el capítulo antes mencionado, se afirma que "Nietzsche está en contra de la esperanza" (Merrick, 2019, p. 101). Es cierto que "Nietzsche está en contra de la esperanza" en la promesa del teísmo, particularmente en la medida en que tal esperanza sirve para pacificar las aspiraciones de uno para este mundo. Pero concluir de esto que Nietzsche está en contra de la esperanza es como concluir de la hostilidad de Nietzsche hacia los efectos de las deudas de gratitud que se perciben como irredimibles hacia un creador abnegado que Nietzsche está "en contra de la gratitud". Si bien Nietzsche no abogaría por la esperanza con exclusión de la agencia, su proyecto filosófico positivo está muy orientado al futuro y en muchos sentidos se caracteriza por la esperanza. Más fuertemente, la postura de Nietzsche sobre la perspectiva de un futuro dionisíaco de la música, la superación del nihilismo, una revalorización de valores, etc., son posiblemente ininteligibles sin entender a Nietzsche como un filósofo de la esperanza.

Pragmatist Hope de Stitzlein (2019) cubre mucho terreno, comenzando con los primeros pragmáticos estadounidenses (Pierce, James, Dewey) y concluyendo con "neo y pragmatistas contemporáneos" (Rorty, Judith Green, Cornel West, Patrick Shade, Colin Koopman). Stitzlein entiende la "esperanza pragmatista" como un "conjunto de hábitos", donde estos hábitos comprenden disposiciones perceptivas, cognitivas y motivacionales para reimaginar creativamente nuestras circunstancias en respuesta a los desafíos encontrados en un mundo no ideal.

A Zen Buddhist Conception of Hope in Enlightenment, argumenta Dunlap (2019): los que identifican la esperanza como un estado orientado al futuro y como tal un obstáculo para la iluminación o un mero trampolín hacia ella, que el budismo zen de Dōgen proporciona una concepción de la esperanza orientada al presente en la que la esperanza es un buen constitutivo de la iluminación propiamente dicha.

Political hope and cooperative community, Stahl (2019) enumera varias funciones que la esperanza puede ocupar en una teoría política y se centra en la posible función justificativa de la esperanza, argumentando que la esperanza de una forma ambiciosa de comunidad (en algo como el sentido empleado por Rawls) puede proporcionar motivos para el respaldo de una sociedad liberal justa.

Hope for material progress in the Age of the Anthropocene, Moellendorf (2019) sostiene que, frente a la destrucción medioambiental provocada por el capitalismo, es sin embargo racional esperar una prosperidad humana generalizada. El argumento de Moellendorf prosigue examinando y modificando el mecanismo identificado por Cohen para explicar la tendencia histórica del crecimiento de las fuerzas productivas. En opinión de Cohen, argumenta Moellendorf, falta la 
apreciación de la contribución hecha por circunstancias ambientales suficientemente favorables, de modo que "cuando los seres humanos trabajan, las mejoras pueden transmitirse al menos a algunas generaciones sucesoras" (p. 253). Moellendorf obtiene apoyo empírico para este "hecho de la favorabilidad climática" (p. 261), como él lo llama, del trabajo en la teoría de la evolución sobre las circunstancias subyacentes al advenimiento de la modernidad conductual y de los datos relacionados con el crecimiento atribuible a la Revolución Industrial. Según Moellendorf (2019), dada la destrucción ambiental atribuible a los factores que subyacen al crecimiento de la capacidad productiva, el crecimiento futuro y la eventual prosperidad generalizada no pueden darse por sentados; sin embargo, pueden esperarse racionalmente. Es decir, es racional esperar que "los hechos de la inteligencia y la racionalidad puedan reunirse para resolver el problema de la destrucción del medio ambiente, que en sí mismo se está convirtiendo en un problema productivo" (p. 263).

Dada su amplitud, es probable que haya algo de valor en el volumen para una amplia gama de lectores de disciplinas que incluyen filosofía, historia, teología y teoría política.

\section{Referencias}

Benton, M. (2019). Epistemological Aspect of Hope. In C. Blöser, \& T. Stahl, The Moral Psychology of Hope (pp. 135-152). Rowman y Littlefield Publishers.

Blöser, C. (2019). Hope in Kant. In C. Blöser , \& T. Stahl , The Moral Psychology of Hope (pp. 57-74). Rowman y Littlefield Publishers.

Blöser, C., y Stahl, T. (2019). The moral psychology of hope. Rowman y Littlefield Publishers.

Cairns, D. (2019). Hope in Archaic and Classical Greek. In C. Blöser, \& T. Stahl, The Moral Psychology of Hope (pp. 15-36). Rowman y Littlefield Publishers.

Dunlap, R. (2019). A Zen Buddhist Conception of Hope in Enlightenment. In C. Blöser , \& T. Stahl , The Moral Psychology of Hope (pp. 209-226).

Fremstedal, R. (2019). Kierkegaard on Hope as Essential to Selfhood. In C. Blöser , \& T. Stahl, The Moral Psychology of Hope (pp. 75-92). Rowman y Littlefield Publishers.

Gallagher, M., D'Souza, J., \& Richardson , A. (2019). Hope in Contemporary Psychology. In C. Blöser, \& T. Stahl, The Moral Psychology of Hope (pp. 189-208). Rowman y Littlefield Publishers.

Jeffrey, A. (2019). Hope in Christianity. In C. Blöser , \& T. Stahl, The Moral Psychology of Hope (pp. 3756). Rowman y Littlefield Publishers.

Martin, A. (2019). Interpersonal Hope. In C. Blöser, \& T. Stahl, The Moral Psychology of Hope (pp. 229248). Rowman y Littlefield Publishers.

Merrick, A. (2019). Historia Abscondita: Or, on Nietzsche, Hope and History. In R. Green, Theories of hope (pp. 99-110). Rowman y Littlefield Publishers. 
Moellendorf, D. (2019). Hope for Material Progress in the Age of the Anthropocene. In C. Blöser, \& T. Stahl, The Moral Psychology of Hope (pp. 249-264). Rowman y Littlefield Publishers.

Snow, N. (2019). Is Hope a Moral Virtue? In C. Blöser, \& T. Stahl, The Moral Psychology of Hope (pp. 171-188). Rowman y Littlefield Publishers.

Snyder, C. (2002). Hope theory: rainbows in the mind. Psychological Inquiry, 13(4), 249-275. https://doi. org/10.1207/S15327965PLI1304_01

Snyder, C., Harris, C., Anderson, J. R., Holleran, S. A., Irving, L. M., Sigmon, S. T., Harney, P. (1991). The will and the ways: development and validation of an individual-differences measure of hope. Journal of personality and social psychology, 60(4), 570. https://doi.org/10.1037/0022-3514.60.4.570

Stahl, T. (2019). Political Hope and Cooperative Community. In C. Blöser, \& T. Stahl, The Moral Psychology of Hope (pp. 265-284).

Stitzlein, S. (2019). Pragmatist Hope. In C. Blöser, \& T. Stahl, The Moral Psychology of Hope (pp. 93-112). Rowman y Littlefield Publishers.

Stockdale, K. (2019). Emotional Hope. In C. Blöser, \& T. Stahl, The Moral Psychology of Hope (pp. 115134). Rowman y Littlefield Publishers.

Vice, S. (2019). Pessimism and the Possibility of Hope. In C. Blöser, \& T. Stahl, The Moral Psychology of Hope (pp. 153-170). Rowman y Littlefield Publishers. 